\title{
A defect determines strength
}

\author{
When you start tearing a piece of aluminium foil apart, you create dislocations in the material. Suhas Eswarappa \\ Prameela and Tim Weihs recount the story of the Burgers vector that is now an indispensable tool for describing \\ dislocations.
}

C rystalline materials, characterized by an ordered and periodic arrangement of their atoms, are all around us, from snowflakes to salts or diamonds. The atoms in these crystals are usually arranged in a lattice. In perfect crystals, atoms occupy ideal, periodic positions, with no disruptions or defects in their arrangement. If all the subatomic particles (for example, electrons) occupied the lowest energy states in such perfect crystals, these would lack most of their electronic, physical and chemical properties $^{1}$ : powdered iron cores used in transformers could not be magnetized without defects in their subatomic structure; silicon would lack its tunable electrical conductivity without atomic defects such as boron or phosphorus. Similarly, we could not form metallic alloys into unique shapes for bridges, cars, or ships without line defects lending them formability.

Imperfections thus bring life to many materials by jostling atoms or sub-atomic particles off their ideal positions. Back in 1934, Egon Orowan, Michael Polanyi and Geoffrey Taylor were trying to explain why the theoretical or ideal strengths of materials are much higher than observed experimentally. To account for this discrepancy, they predicted what was then a new class of line defects or imperfections called dislocations ${ }^{2}$, which occur when there is an abrupt change in the arrangement of a row of atoms that affects translational order. The defect is analogous to a bone dislocation that we see during injuries to a joint, but a billion times smaller.

During the early stages of dislocation theory, the Dutch physicist Jan Burgers derived a simple but powerful way to indicate the type and magnitude of dislocations a vector that is named after him, the Burgers vector $^{3}$. But it took nearly two decades before dislocations were observed directly, in experiments with aluminium ${ }^{4}$ using a transmission electron microscope.

If you were to travel a square path in a perfect cubic crystal, moving equal atomic distances along each side, you would end up

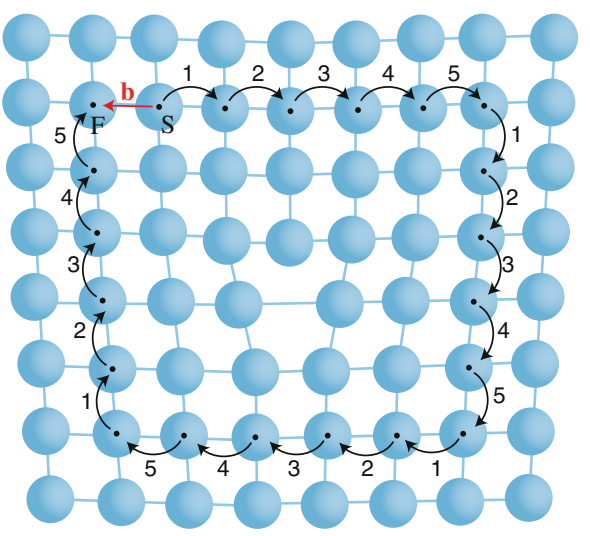

back where you started. This is no longer true if the crystal contains a dislocation, as pictured; here, the dislocation in the centre of the cubic aluminium crystal extends in and out of the page's plane. In this case, the equiatomic path around the line defect yields a gap between the start $(\mathrm{S})$ and finish $(\mathrm{F})$ points - the vector connecting them is called the Burgers vector $\mathbf{b}$. Its magnitude quantifies the displacement or deformation that is enabled by the movement of a single dislocation across the crystal, and it is determined by the lattice spacing between atoms for a given crystal and the atomic planes on which the dislocations move. Given that the magnitude of the Burgers vector typically ranges between one and three ångströms, many millions of dislocations are required to enable crystal deformation that is visible to the human eye. For example, when we tear off a piece of aluminium foil to cover a dish in our kitchen, we force millions of dislocations to glide across the small crystals in the foil near the torn surface, the motion of each providing a displacement of approximately two ångströms.

Today, the Burgers vector is used to describe how crystalline defects determine the properties of materials and is related to the deformation, fatigue, and fracture of materials. In terms of deformation, when bulk mechanical stresses drive a crystal to deform, the macroscopic forces on the crystal can be related to the nanoscale forces on individual dislocations with the help of the Burgers vector. With advanced computational tools such as dislocation dynamics, the movement of hundreds of dislocations in a crystal can be predicted, gaining insights into how other defects such as solute atoms, crystal boundaries, and intermetallic particles can inhibit their motion. This, in turn, enhances our ability to design durable and robust materials such as aluminium alloys for use in automobiles and aircraft.

The Burgers vector is also employed when exploring material properties beyond strength and failure: phonons are scattered by dislocations, and the Burgers vector is used heavily in formulating drag mechanisms. Dislocations are also important to electronic materials and the semiconductor industry. They can explain the growth of strained lattices or patterned structures in semiconductors and the failure of transistors because dislocations act as scattering centres for electrons and holes. Burgers's description of dislocations within a crystal has moved well beyond its original intent of explaining material strength and continues to prove useful in designing materials for applications ranging from the electronic chips in mobile phones to the advanced metallic alloys ${ }^{5}$ in aircraft engines and construction.

Suhas Eswarappa Prameela (D) 1,2凶 and Timothy P. Weihs ${ }^{1,2}$

${ }^{1}$ Department of Materials Science and Engineering, Johns Hopkins University, Baltimore, MD, USA.

${ }^{2}$ Hopkins Extreme Materials Institute, Johns Hopkins University, Baltimore, MD, USA.

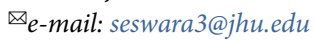

Published online: 7 July 2020

https://doi.org/10.1038/s41567-020-0961-2

References

1. Cai, W. \& Nix, W. D. Imperfections in Crystalline Solids (Cambridge Univ. Press, 2016).

2. Hull, D. \& Bacon, D. J. Introduction to Dislocations (Elsevier, 2011).

3. Burgers, J. M. Proc. Phys. Soc. 52, 23-33 (1940).

4. Hirsch, P. B., Horne, R. W. \& Whelan, M. J. Philos. Mag. J. Theor. Exp. Appl. Phys. 1, 677-684 (1956).

5. Prameela, S. E. et al. Materialia 9, 100583 (2019).

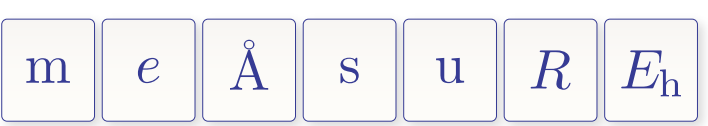

\begin{tabular}{|l|l|l}
\hline$\mu_{0}$ & $\varepsilon_{0}$ &
\end{tabular} $\mathrm{R} \mathrm{e}$

